\title{
LAS CIENCIAS DE LA EDUCACIÓN. ENTRE UNIVERSALISMO Y PARTICULARISMO CULTURAL*
}

\author{
Rafael Ríos Beltrán \\ Universidad de Antioquia, Colombia
}

\section{INTRODUCCIÓN}

Quien se interese hoy en día por las ciencias de la educación se encuentra con un campo disciplinar y profesional (Hofstetter y Schneuwly, 2001, 7-20) de diversas configuraciones dependiendo de las particularidades propias de cada cultura. De ahí que analizar las ciencias de la educación desde una perspectiva comparada no sea una tarea fácil. Bien sabemos que la educación comparada es todavía una espacio por conquistar, un eslabón perdido y débil.

Existe una tendencia muy marcada en algunos estudios comparados de examinar solamente lo semejante, haciendo caso omiso de las particularidades culturales. Por ejemplo, "todos los investigadores en ciencias de la educación han minimizado el papel que puede jugar la consideración de las diferencias, así como el de las reivindicaciones regionales en las políticas de democratización y de igualdad de oportunidades". (Furter, 1996, 95)

Pero, si a "la manera de una mujer que va a comprar lana y compara dos madejas que sospecha que no son del mismo color" ( Veyne,1976) se compara un sistema educativo con otro, se hace para constatar que son no solamente semejantes sino también diferentes. Por eso, las descripciones históricas que el presente escrito intenta realizar sobre las ciencias de la educación para futuros estudios comparados no busca “..reducir la diversidad de los discursos y a dibujar la unidad que debe totalizarlos"; sino a "repartir su diversidad en figuras diferentes". (Foucault, 1996, 268) En efecto, en un análisis comparado se debe hacer visible la diversidad y disparidad que existen en el interior de una misma unidad territorial.

Ya que en Suiza no hay denominación común de ciencias de la educación, ni de pedagogía, por sus características "asombrosamente heterogéneas" (Hofstetter y Schneuwly, 2001, 50) describiremos, especialmente, el Cantón de Ginebra. Allí desde 1920, existe una formación universitaria completa en ciencias de la educación con mención en pedagogía.

\section{1. el estatuto de la pedagogía: avatares Y CONDiciones De EXISTENCIA}

Para Daniel Hameline:

"El siglo XIX - y particularmente su segunda mitad, en el cual todos los paises desarrollados junto con la escuela popular toman su carácter de moderna - puede ser llamado, en verdad, la edad

\footnotetext{
"Este artículo son resultados del proyecto de investigación: "El saber pedagógico y las ciencias de la educación". Financiado por el Comité de Investigaciones (CODI) de la Universidad de Antioquia - Colombia y la Escuela Normal Superior de Copacabana - Colombia.
} 
de Oro de la pedagogia. [...] El siglo XX, será entonces la era de las ciencias de la educación. El paso del singular al plural no es un simple hecho lingüístico. Señala un cambio a la vez epistemológico y societal". (Hameline, 2002, 591)

Pero esta Edad de Oro de la pedagogía y posterior desplazamiento por las ciencias de la educación, no se efectúo de manera natural, ni semejante en cada una de las culturas. Por ejemplo, en Francia difícilmente podemos hacer visible una época dorada de la pedagogía, cuando desde el siglo XIX la pedagogía, según las expresiones de Compayré, "gozaba de respeto en otros países, sin embargo, a los franceses no les suena bien a los oídos la palabra pedagogía. Se le empleaba con cierta repugnancia." (Best, 1972, 58). Como veremos a continuación, el siglo XIX y parte del siglo XX en Francia fue pobre en cuanto a la apropiación de la pedagogía en el ámbito institucional. En consecuencia, el estatuto de la pedagogía como saber y disciplina para la formación de maestros siempre fue cuestionada, desvalorizada, o en el peor de los casos considerada como saber menor y restringido solamente a los métodos.

En 1810, se crea en Estrasburgo la primera Escuela Normal Primaria en Francia, inspirada más en los seminarios pedagógicos alemanes que en la Escuela Normal de París creada por la Convención revolucionaria en 1794. La Escuela Normal Primaria de Estraburgo se convirtió en el modelo administrativo ${ }^{1}$ para las nacientes Escuelas Normales Primarias de la República, abiertas progresivamente por todo el territorio francés mediante la Ley Guizot del 14 de diciembre de 1832, la Ley Duruy del 10 de abril de 1867 y por la ley emitida por Jules Ferry el 9 de agosto de 1879. En ellas se enseñó durante algún tiempo ${ }^{2}$ la pedagogía entendida solamente desde el punto de vista de los métodos para perfeccionar el arte de enseñar a leer, escribir y contar.

La pedagogía como disciplina y saber sólo tuvo un lugar marginal durante el ministerio de Jules Ferry. A través del Decreto de 22 de julio de 1881 se introdujo en las Normales un curso de pedagogía y administración escolar, al cual solo se le dedicaba una hora semanal en los primeros dos años de estudios y una y media en el tercer y último año. (Buisson, 1911) Mientras que en otros regiones europeas su enseñanza era mucho más intensiva: en Baviera ocupaba 5 horas en el primer año y 4 en el segundo; en Austria 3 horas en el segundo año, 5 en el tercero y 9 en el cuarto. (Dottrens, Mialaret, 1971, 58)

La introducción de la pedagogía en las Escuelas Normales Superiores fue más tardía y se inició con algunas experiencias institucionales efímeras.(Gautherin, 1991, 129) El primer curso de pedagogía fue instituido en 1847 por Salvandy, ministro de la instrucción pública y por Dubois director de la Escuela Normal Superior. Fue confiado al profesor del Colegio Real de Bourdeaux, Charles Thurot, quien no contaba con conocimientos en pedagogía. Fue elegido por su erudismo, prudencia, buena conducta y por el conocimiento de varias lenguas lo cual le habilitaba para seguir la historia de la educación. El curso fue un gran fracaso, del cual el director de la escuela normal, G. Perrot, hablaba todavía en 1899 en su testimonio frente a la comisión de investigación sobre la enseñanza secundaria: "este curso fue hecho por un hombre muy distinguido y él mismo sintió su inutilidad, M. Thurot, mi colega de la Academia de las Inscripciones; fue él mismo quien pidió ser encargado, en lugar del curso de pedagogía, de la conferencia de gramática que hizo muy bien." (Gautherin, 1991, 127)

\footnotetext{
${ }^{1}$ Pues el modelo pedagógico fueron las escuelas cristianas de Juan Bautista de la Salle

${ }^{2}$ En especial, durante las tres primeras décadas del siglo XIX hasta que con Guizot la formación pedagógica de los maestros es acompañada con otras materias.
} 
Como se puede apreciar, fueron evidentes las resistencias de la Escuela Normal Superior de París a la introducción de cursos de pedagogía. Para su director Emest Bersot, se trataría de una idea inmadura, pues con el conocimiento de su disciplina el profesor de secundaria estaría habilitado para enseñar. En 1878, Bersot afirmaba:

"cualquiera que sea la materia, no hay dos formas de enseñar: en gramática, el firme conocimiento de las leyes gramaticales, la precisión de la explicación y de la traducción de los textos; en letras con el mismo fondo indispensable, el destello literario; en historia la posesión de los hechos, los juicios críticos, el talento de exponer, eligiendo, alejando, poniendo cada cosa en su lugar y dándole su valor...." (Gautherin, 1991, 128)

De manera similar, para el historiador Numa-Denys Fustel de Coulange, nuevo director de la Escuela luego de la muerte de Bersot, la pedagogía sería un aprendizaje del cual podía prescindir el futuro profesor:

"Al profesor, algunas nociones de pedagogía bastaban sobre todo cuando había hecho estudios psicológicos. El actúa sobre sus alumnos y toma el mando sobre ellos por los conocimientos que les enseña; no tiene que recurrir a medios artificiales. Que él ama la verdad y les muestra su sabor, es bastante como para que sea maestro de sus alumnos. En la Escuela Normal no se enseña la pedagogía, y no deseo que se la enseñe. (...) sin ninguna teoría pedagógica, nosotros les enseñamos durante tres años a amar la ciencia, y solamente con esto serán buenos profesores." (Gautherin, 1991, 130)

Habría que esperar la década de los ochenta del siglo XIX para que se diera una primera institucionalización de la pedagogía en las instituciones formadoras de profesores de secundaria. En primer lugar, con la creación en 1879 de la primer Escuela Normal Superior de Chicas establecida en Izeure y luego trasladada a Fontenay-aux-Roses. En esta Normal ocupó un lugar importante la psicología y la moral aplicadas a la educación, la historia y la crítica de las doctrinas y métodos pedagógicos. Años después, siguiendo el mismo modelo, se crearía la Escuela Normal Superior de Chicos, inicialmente en Sévres y luego trasladada a Saint-Cloud. (Vial, 1982, 62-63) Por su parte, en la Escuela Normal Especial de Cluny ${ }^{3}$, los futuros maestros de la enseñanza especial eran aquí formados y preparados para una agregación específica. El curso de pedagogía fue dado entre 1866 a 1872 por Ferdinand Roux, director de la escuela. Pero en 1872, acusado de incompetencia administrativa, científica y financiera, Roux fue dimitido de sus funciones y el curso de pedagogía $a^{4}$ fue clasurado.

Durante el periodo comprendido entre 1883 y 1914, la pedagogía en Francia se abre paso más allá de las Escuelas Normales Primarias y de las Escuelas Normales Superiores, incursionando en las Facultades de Letras de las universidades como cátedra universitaria. Paradójicamente, Ferdinand Buisson profesor de la cátedra de pedagogía o ciencia de la educación en la Sorbonna, desde 1896 hasta 1902, se refería así a la pedagogía en su Lección Inaugural de 1896: "No se humilla la pedagogía recordando que esta solo puede ser una ciencia de aplicaciones, y aplicaciones de segunda mano". (Buisson, 1896, 502).

Con Durkheim, en las cátedras de pedagogía en la Sorbonna, el estatuto de la pedagogía encontrará desde la sociología y su relación con la ciencia de la educación unas condiciones de existencia distintas. Para él, la pedagogía se encuentra en un punto intermedio entre el arte y la ciencia. No es arte, pues el arte "está referido a todo lo que es la práctica pura sin teoría" (Durkheim, 1912, 1539) De esta manera, Durkheim

\footnotetext{
${ }^{3}$ La Escuela de Cluny fue creada por Victor Duruy en agosto de 1865.
} 
intenta reorganizar el discurso y la institucionalidad de la pedagogía, pero no le proporciona a esta un discurso que le sea propio, sino que la deja dependiendo totalmente del desarrollo de la ciencia de la educación. Además, la pedagogía como "teoría práctica" sería tan sólo una "aplicación" de las nociones que desarrolle esta ciencia, quedando imposibilitada de construir sus propios conceptos. (Zuluaga, Sáenz, Ríos, Herrera, 2004)

Las cátedras universitarias sobrevivieron hasta 1914, momento en el cual son suprimidas progresivamente, para desaparecer definitivamente en 1918. En consecuencia, entre 1918 y 1957, la pedagogía difícilmente se asentó como institución. Ella se refugió nuevamente en espacios como los cursos prácticos de las Escuelas Normales Primarias ${ }^{5}$, en las clases de pedagogía dictadas en la Sorbona entre 1945 y 1957 por Roger Cousinet, difusor de las ideas sobre la Escuela Nueva en Francia desde 1921, en el Instituto de Pedagogía abierto en 1920 y adscrito a la Facultad de Letras de la Sorbona, y en la Escuela Práctica de Psicología y de Pedagogía, creada en Lyon, en 1945, bajo la dirección de Bourjade.

En Ginebra-Suiza, tampoco nos encontraremos con aquella época "dorada" vivida por la pedagogía, según Hameline, en el siglo XIX. Curioso acontecimiento en una sociedad que la Historia de la Pedagogía y la Educación, se ha encargado de mostrarla como la "Meca de la pedagogía". Aunque las condiciones de existencia y de relación con el plural ciencias de la educación son distintas y menos subalternas que en la cultura francesa, la pedagogía no encontrará su real "Edad de Oro". Según Hofstetter y Schneuwly, el proceso de institucionalización y disciplinarización de la pedagogía en la Universidad de Ginebra entre los años de 1880 y 1916, no significó su autonomía real. (Hofstetter, Schneuwly, 1997, 149)

Por e contrario, circulaba bajo la dependencia de la administración pública, de las concepciones moralistas, filosóficas, patrioticas y psicológicas. (Hofstetter, Schneuwly, 1997, 150) Condición de existencia que no le permitió escapar de ser tratada como disciplina secundaria entre los estudiantes de Letras, ciencias sociales y ciencias.

Así como en Francia todos los intentos relacionados con la autonomía, la disciplinarización y la institucionalización de la pedagogía en los espacios universitarios fueron desacreditados y ocuparon un lugar secundario, precisamente porque su "enseñanza se sumaba la mayor parte del tiempo a la enseñanza de la filosofía, de la psicología (en el caso de Foucault) o de la sociología (en el caso de Espinas, de Durkheim, de Bouglé ou de Fauconnet)" (Gautherin, 1995, 47), también, en Ginebra algunos intentos para dinamizar la pedagogía y proporcionarle una nueva orientación fueron cuestionados y desplazados. Hofstetter y Schneuwly afirman que en Ginebra, a principios del siglo XX, se vivió una gran efervescencia pedagógica en el ámbito universitario, a través de cuatro intentos que perseguían darle unas condiciones de existencia distintas a la pedagogía. $(2001,88)$

El primer intento esta relacionado con la creación en 1906 de un seminario de pedagogía experimental. En este momento se pretendía reorientar la pedagogía hacia lo experimental por medio de los trabajos de psicología experimental aplicados a la educación del médico Edouard Claparède. En 1909, aparece en el

\footnotetext{
${ }^{4}$ Por el trabajo de tesis de Gautherin (1991) sabemos que en la escuela de Cluny la pedagogía era una "materia" del programa, que a diferencia de las otras -incluso de la moral- no exigía competencias científicas ni profesionales particulares.

${ }^{5}$ Las cuales fueron suprimidas en 1940 por el gobierno de Vichy. En 1947 fueron nuevamente restablecidas.
} 
escenario universitario, el segundo intento por transformar la pedagogía, esta vez con Lucien Cellérier y Jules Dubois al proponer un Seminario de Pedagogía con el objetivo de desarrollar, mediante conferencias, aspectos sobre Historia de la pedagogía, de Pédología, de psicología, de filosofía y de moral pedagógica. El tercer intento, surge en 1912, cuando la politica se interesa por introducir un sentido utilitario a la pedagogía, es decir, un rol más directo con lo empírico, la formación de maestros y la eficacia de los sistemas de formación. En este mismo año, y en el contexto de la creación de la Facultad de Ciencias Económicas y Sociales, surge el cuarto intento por renovar la pedagogía en la Universidad de Ginebra. Aquí se pensaba darle a la pedagogía su condición de ciencia social. Pero, éstos cuatro intentos se frustaron por la presiones surgidas en Ginebra a nivel político, institucional y epistemológico para no permitir la formación de los maestros, ni la constitución de la pedagogía como ciencia experimental y social en el ámbito universitario. (Hofstetter y Schneuwly 2001, 8892)

Sin embargo, los efectos de ésta debatida y frustrada efervescencia pedagógica en Ginebra, se orientaron hacia la creación, en 1912, de un Instituto llamado "Ciencias de la Educación". Así lo afirma Claparède al exponer las necesidades a las cuales responde el Instituto de Ciencias de la Educación:

"El proyecto de un Instituto de este genero deriva de una doble constatación: de una parte, la preparación psicológica y pedagógica de los maestros es insuficiente, de otra parte, ninguna medida ha sido tomada para asegurar los progresos y desarrollos de la ciencia de la educación. Estas dos lagunas son las que nuestro instituto pretende reformar y mejorar". (Claparède, 1912, 5)

Como se puede apreciar, el plural ciencias de la educación surge en Ginebra en 1912, para renovar las condiciones de existencia de la pedagogía o ciencia de la educación, y la débil formación pedagógica y psicológica de los maestros en el ámbito universitario durante la segunda mitad del siglo XIX e inicios del siglo $X X$.

La era de las ciencias de la educación se inicia en Ginebra, entonces, para ocuparse de la psicología del niño y de pedagogía experimental, ajustando las técnicas del educador a los conocimientos psicológicos. (Piaget, 1999, 190) En este caso, la pedagogía no sale excluida por las ciencias de la educación, como le sucedió en la experiencia francesa, sino cobra existencia con el nombre de "pedagogía experimental" y bajo la tutela de la psicología de corte experimental. Por lo que se ve, "no se trata de un fenómeno característico del área lingüística y cultural francófona en absoluto, sino más bien de un fenómeno peculiarmente francés." (Schriewer, 1991, 165)

En Colombia durante el siglo XIX, la única Edad de Oro conocida no es precisamente la de la pedagogía, sino la del movimiento de reorganización del sistema educativo colombiano conocido como "La Reforma Instruccionista de 1870" Este movimiento liderado por los "Liberales Radicales" fue un rotundo fracaso, a causa de la guerra civil de 1876, la crisis económica interna y la deuda externa. (Saldarriaga, 2004, 240).

Mediante el decreto 2 de noviembre de 1844, la pedagogía en Colombia tiene su condición de existencia, tanto discursiva como institucional, en las Escuelas Normales. En las Universidades y Colegios no existían cátedras para la formación de maestros. (Zuluaga, 1984, 80, 91) Las escuelas normales fueron creadas en Colombia mediante Ley 6 de agosto de 1821. El decreto 20 de enero de 1822 las sitúa en las ciudades de Bogotá, Caracas y Quito, donde se aplicaría el método de enseñanza mutua creado por el inglés José Lancaster. 
Pero es con el Decreto 2 de noviembre de 1844 que la Escuela Normal se transforma de escuela primaria, dedicada al entrenamiento en la enseñanza mutua desde 1822, en una institución formadora de maestros y apoyada en los principios y la práctica de la pedagogía. La pedagogía institucionalizada en 1844 en las Normales fue la pedagogía pestalozziana, objetiva o intuitiva, mediante el Manual de enseñanza mutua para las escuelas de la primeras letras, elaborado por José Maria Triana en 1845. Este Manual contenía otros tres manuales: el primero desarrollaba el programa de pedagogía, y en los otros dos algunos elementos del método de Pestalozzi para la gramática y la aritmética adaptados para la instrucción primaria. De acuerdo, con Zuluaga, "los contenidos de estos manuales se constituyeron en el nuevo saber pedagógico, el cual se diferenciaba del saber pedagógico anterior en cuatro aspectos: los conceptos de pedagogía y educación, los fines de la educación, el papel del método en la educación y la instrucción, el nuevo carácter del maestro".(Zuluaga, 2001, 46)

En otras palabras, desde 1844 la Escuela Normal se convierte en la "base institucional" de la pedagogía en Colombia y en una institución de saber formadora de maestros. (Zuluaga, 2001, 41) No obstante, con la Ley 15 de mayo de 1850 sobre Libertad de enseñanza, las condiciones de la Escuela Normal como institución de saber formadora de maestros comienza a ser cuestionada por los liberales bajo la convicción que ésta institución podía influir en la formación de un gremio, en este caso de los maestros. (Zuluaga, 1999, 428) De esta manera, la Normal es cerrada en 1851 por José Maria Plata, Gobernador de la Provincia de Bogotá entre 1850 y 1851 . Esta decisión fue confirmada por su sucesor Patrocinio Cuellar, al explicar en su informe como Gobernador en 1853 que la Escuela Normal se había quedado sin objeto. En consecuencia, la Escuela Normal salió del escenario de la legislación sobre instrucción primaria desde 1853 hasta la sanción del Código de Instrucción Pública en 1858 que le dio nuevas esperanzas a la Escuela para su reapertura. Sin embargo, los avatares políticos, la guerra y sus efectos retardaron la aplicación del Código. Sólo hasta el Decreto 1 de noviembre de 1870 la Escuela Normal retomó su condición de institución de saber formadora de maestros.

Así , desde 1872, la pedagogía pestalozziana u objetiva "empieza a ser difundida oficialmente en las normales establecidas en cada una de las capitales de los Estados Unidos de Colombia, a cargo de los maestros protestantes de la primera Misión Pedagógica Alemana, traída al pais por los gobernantes liberales radicales. Esta vez ya no aparecía como un simple procedimiento, sino que fue proclamada como un método universal, teórico y práctico, para la "reforma de la mente de la niñez colombiana". (Sáenz, Saldarriaga, Ospina, 1997, 13) Los acontecimientos bélicos de 1876, acabaron con este primer proyecto nacional de educación, pero el método de enseñanza quedó incluido al sistema de educación pública, bajo el lema de oro del método pestalozziano: "cosas, antes que palabras" hasta el año de 1932, cuando el Gobierno Nacional de Colombia se vio en la necesidad de clausurar la casi totalidad de las Escuelas Normales, excepto la de Medellin, Tunja y Bogotá, por la escasez de fondos para su sostenimiento y la carencia de personal competente para dirigirlas y regentar las cátedras correspondientes.

En este contexto, surgen las primeras Facultades de Ciencias de la Educación en Colombia, mediante Decreto 1990 de 1933. La primera surge en 1933, al interior de la Universidad Nacional de Colombia en Bogotá, la segunda en 1934 en el Instituto Pedagógico de Señoritas, y la tercera en Tunja, también en 1934. En estas tres facultades existió la pedagogía como sección de estudios o especialidad, y no como cátedra como fue el caso para Suiza y Francia. 
Con el surgimiento de las Facultades de Ciencias de la Educación en Colombia, por un lado, se comienza la preparación universitaria de los maestros y se introducen los estudios en pedagogía en el ámbito universitario; y por el otro, los saberes y las prácticas pedagógicas comienzan a cambiar y a transformarse sobre un nuevo suelo epistemológico: el experimental Por su parte, la episteme clásica sustentada en la Lógica y la Gramática y suelo epistemológico originario de la pedagogía pestalozziana se desplazó progresivamente en el saber pedagógico colombiano.

Pero la existencia de la pedagogía en Colombia en el espacio universitario, durante la primera mitad del siglo XX, y como sección de estudios especializados en las Facultades de ciencias de la educación, comienza su fin, en primer lugar, cuando las tres facultades de ciencias de la educación, la de varones de Bogotá y Tunja y la de señoritas en el Instituto Pedagógico fueron fusionadas en una sola mediante Decreto 1917 de octubre 25 de 1935, y en segundo lugar, con la Ley 39 de febrero 21 de 1936, la cual dispusó que la Facultad fusionada de Ciencias de la Educación continuará funcionando con el nombre de Escuela Normal Superior bajo la tutela del Ministerio de Educación Nacional e independiente de la Universidad Nacional.

Con la creación de la Escuela Normal Superior de Colombia, como reemplazo de la Facultad de Ciencias de la Educación, la pedagogía fue suprimida como sección de estudios especializados y pasó a ser considerada como "un conjunto de métodos y técnicas para estimular el desarrollo físico e intelectual de niños y jóvenes, impartirles los conocimientos y facilitarles la adquisición de los mismos". (Socarras, 1987, 33) De esta manera, la nueva institución formadora de maestros inclinó más sus preocupaciones por las ciencias humanas y sociales que por constituir la Sección de Pedagogía, en un campo autónomo, conceptual, plural y abierto. Por su parte, directores y estudiantes de la desaparecida Facultad en Bogotá argumentaban que el cambio de nombre y dependencia le restaba status de educación universitaria a la formación de los maestros de secundaria. Vale la pena señalar que en Colombia la pedagogía era un saber sometido, no era reconocida. Pero desde 1980, el Grupo de investigación Historia de la práctica pedagógica en Colombia, "a partir del análisis de los pedagogos clásicos y algunos contemporáneos, y a partir de conocer los avatares de las condiciones de existencia del maestro, fue la erudición como crítica la que permitió al grupo, encontrar tensiones y posibilidades en el saber pedagógico, y evidenciar las relaciones de saber y poder que se daban entre los sujetos, (maestro y alumno), discursos e instituciones". (Zuluaga, 2004)

Como podemos apreciar en estas tres culturas, los avatares de la pedagogía, a nivel discursivo e institucional, durante el siglo XIX y parte del siglo XX, difícilmente nos permite hacer visible la Edad de Oro en la pedagogía. Por el contrario, nos muestran las condiciones de dependencia, desplazamiento y fragmentación, en las cuales ha existido la pedagogía. En otras palabras, algunas prácticas culturales, especificadas en este escrito, han producido unas condiciones - o por lo menos, una cultura académica particularmente adversas a la existencia de un campo de saber, de reflexión y práctica sobre la pedagogía. En consecuencia, a éstas tres culturas, las atraviesa su relación tensionante con el discurso de la pedagogía. Tal condición singular ${ }^{6}$ abrió, no solamente a nivel universal sino también particular, debates terminológicos que desembocaron en debates y reflexiones de tipo epistemológico, los cuales desarrollaremos en el siguiente aparte de este escrito.

\footnotetext{
${ }^{6}$ Con esta expresión quiero decir lo que tienen de particular, especial y raro estas tres culturas, lo particular no es lo común, precisamente es apartarse de lo común.
} 


\section{DE LA PEDAGOGÍA O CIENCIA DE LA EDUCACIÓN A LAS CIENCIAS DE LA EDUCACIÓN. EL DEBATE EPISTEMOLÓGICO}

Como se ha visto la pedagogía ha sido, cultural e históricamente, un campo de debate: un lugar de tensiones y luchas, en el cual los debates se han dado en torno a su legitimidad científica; a sus posibilidades de existencia como campo de saber autónomo o como disciplina. Y esto, porque se trata de un "saber liminal; eso es, un saber que se sitúa en las fronteras - o mejor en los intersticios - problemáticos del pensamiento, los saberes y las prácticas modernas”. (Zuluaga, Sáenz, Rios, Restrepo, 2004) Para decirlo de otra manera, la pedagogía se podría considerar, históricamente, como un "saber sometido", es decir, como un saber descalificado, no competente e insuficientemente elaborado, saber ingenuo, jerárquicamente inferior, por debajo del nivel de conocimiento o cientificidad requerido. (Foucault, 1992, 21)

En estas condiciones, se produce el paso de la ciencia de la educación o pedagogía a las ciencias de la educación durante el siglo XX. Puente que no significó un cambio de nombre solamente, ni según Mialaret y Dottrens, "el efecto de una moda pasajera: corresponde al desarrollo de todas las disciplinas que están en relación con la educación. No estamos ya en los tiempos en que los conocimientos del "pedagogo" habían de ser forzosamente rudimentarios. La formación de un especialista en ciencias de la educación exige numerosos años y competencias muy variadas". (Dottrens, Mialaret, 1972, 45)

Los años de 1912, 1933 y 1967 significaron el comienzo de la era de las ciencias de la educación en Ginebra-Suiza, Colombia y Francia, respectivamente. Tales inicios significaron, el desplazamiento de la pedagogía como cátedra, disciplina académica y como sección de estudios, en el ámbito universitario. Pero también, significó el inicio de los debates y reflexiones epistemológicas que se dirigieron a oponer una pedagogía considerada como precientífica, ambigua, ingenua e incompetente con un conjunto de ciencias, llamadas "ciencias de la educación", que abordan los problemas educativos desde múltiples objetos y metodologías, provenientes de las jóvenes ciencias humanas. Acontecimiento epistemológico que tiene poco o nada de singular en éstas tres culturas, lo cual hace que las reflexiones y debates epistemológicos sobre las ciencias de la educación se caractericen por su hibridismo ${ }^{7}$.

Francia es la cultura que ha desarrollado las más múltiples reflexiones epistemológicas, alrededor de la identidad de las ciencias de la educación, y los más intensos debates sobre la existencia disciplinar y científica de éstas ciencias al interior de las Universidades Francesas. Desde 1967 una parte importante de los investigadores en ciencias de la educación se ocupa del debate existencial: ¿ existen las ciencias de la educación?, ¿por qué no pedagogía y sí ciencias de la educación?, ¿deben existir en el ámbito universitario?, ¿cuáles son sus objetos y métodos?, ¿cuál es su especificidad? ¿Por qué tienen dificultades para existir? Así lo confirma Avanzini al afirmar que: "No hay un Congreso, un Coloquio, un encuentro en Ciencias de la Educación, donde el problema de la especifidad de nuestra disciplina no sea planteado". (1992, 119). Diferentes obras representadas en artículos y libros sostienen esta línea de discusión en la cultura francesa.

El paso del singular al plural se produce en Francia, en 1967, con la creación de los estudios en Ciencias de la Educación en las Facultades de Letras. El surgimiento del plural materializó por completo, el

\footnotetext{
${ }^{7}$ Al usar el término "hibridismo" no pretendo oponer sino hacer visible el cruce o andamiaje de discursos que unen al mismo tiempo, lo universal y lo particular con respecto a las ciencias de la educación.
} 
efecto que produjo el estatuto desvalorizado de la "pedagogía" en Francia durante la primera mitad del siglo XIX e inicios del siglo XX. Las siguientes expresiones de Debesse dan cuenta de ello:

"Si yo propuse y obtuve que se utilizara la expresión ciencias de la educación al nivel de la enseñanza universitaria, más bien que hablar de pedagogía no ha sido por detestar esta vieja palabra - para substituirle, por una usurpación ridícula, un título más deslumbrante. Es debido a que la palabra pedagogía se volvió doblemente equivoca al mismo tiempo que es demasiado limitante y a la vez demasiado imprecisa" (Debesse, 1976, 71)

Actualmente, para algunos franceses como Michel Develay (2001) y Jacques Gautherin (1995) la existencia de cátedras de pedagogía o de ciencia de la educación, dictadas por Henri Marion, Ferdinand Buisson, Thamin, entre otros, en las universidades francesas desde 1883 hasta 1914, no representan ninguna continuidad, ni son un acontecimiento anunciador de las ciencias de la educación. (Develay, 2001, 11)

Sin duda, las ciencias de la educación en Francia existen institucionalmente, no obstante, los debates actuales sobre la pedagogía y el estatuto disciplinario de las ciencias de la educación, tres décadas después de su institucionalización en las universidades francesas (1967), dejan ver que se trata de un campo todavía en disputa y sobre el cual no se han logrado unos consensos mínimos desde el punto de vista epistemológico. Así lo demuestran muchas de sus reflexiones sobre su estatuto, las cuales circulan por diferentes opiniones. (Mialaret, 1977, Ardoino,1977, Meirieu, Develay, 1992, Meirieu, 1995, 1998, Charlot, 2001, AECSE, 2001, Marcel, 2002)

En Suiza, y de acuerdo con los documentos consultados, los debates epistemológicos son de menor intensidad y de poco interés por desarrollarlos. Así lo demuestran las expresiones del profesor de la Universidad de Ginebra Bernard Schneuwly: "el problema es saber lo que queremos hacer con estas ciencias de la educación. No son solamente los objetos científicos que se construyen, sino también las ciencias mismas. Produzcamos investigaciones, apoyándonos sobre esta cultura específica que se desarrolló en "ciencias de la educación", pues es a partir de estas investigaciones efectivas (y no de discursos sobre lo que las ciencias de la educación son "por naturaleza" y deben ser) que se podrá avanzar sobre la cuestión de saber si las ciencias de la educación tienen o no una especificidad epistemológica - y cuál". (Charlot, 2001,153)

Precisamente, Bernard Schneuwly y Rita Hofstetter, responsables del "Equipo de investigación en Historia de las ciencias de la educación" (ERHISE) en la Universidad de Ginebra, abren las reflexiones en torno a las ciencias de la educación en una perspectiva que asocia la cuestión epistemológica, la aproximación histórica y la reflexión sobre la institucionalización. Sus investigaciones se inclinan por estudiar la emergencia y desarrollo de las ciencias de la educación desde el punto de vista de la disciplinarización en los siglos XIX y $X X$.

Inspirados en los recientes trabajos de historia y sociología de las ciencias que tienen por objetivo describir y comprender el desarrollo de las ciencias a través del análisis minucioso de sus prácticas productoras de conocimientos científicos, de su progresiva profesionalización e institucionalización académica, de las transformaciones y controversias cognitivas y socioprofesionales que acompañan su desarrollo, el equipo de investigación (ERHISE) de la Universidad de Ginebra ha venido desarrollando reflexiones sobre las ciencias de la educación en el ámbito regional, (Ginebra) nacional, (Universidad de Bâle, Berne, Laussane, Zurich) e internacional (Alemania, Bélgica, Estados Unidos, Francia, Inglaterra, Italia, Brasil, Portugal, Finlandia, Esapaña...). (Schneuwly, Hofstetter, 2000, 53) 
Desde esta perspectiva, las ciencias de la educación tienen su existencia en Suiza en el cruce de dos campos: el campo profesional y el campo disciplinar. Entre éstos dos campos, dinámicos y extrechamente ligados, encontramos los procesos de disciplinazación de las ciencias de la educación y sus formas de existencia y de desarrollo. (Hofstetter, Schneuwly, 2001, 2002)

En Colombia los debates y reflexiones giran en torno a la imposibilidad de hacer reflexiones histórico-epistemológicas porque ni la pedagogía, ni las ciencias de la educación, tienen un objeto y un método específico para la construccción de un discurso científico. Tanto la pedagogía como las ciencias de la educación "carecen de un discurso científico que nos permita aplicar el método de la historia epistemológica tal como lo plantea Georges Canguilhem: la recurrencia, entendida como jurisdicción crítica sobre el pretérito de un presente científico". (Zuluaga,1999, 59)

Los análisis se inclinan por hacer visible, desde una perspectiva histórica, arqueológica y genealógica, los procesos de disciplinarización de la pedagogía que de las ciencias de la educación. ¿ Por qué sí elegir la pedagogía y por qué no las ciencias de la educación? En primer lugar, porque la pedagogía es "la disciplina que conceptualiza, aplica y experimenta los conocimientos referentes a la enseñanza de los saberes específicos, en diferentes culturas." (Zuluaga, 1999, 11) Ésta definición subraya la especificidad de la pedagogía como un campo de saber que tiene su propia historia, conceptos, prescripciones y objetos de saber, el cual tiene un discurso propio, un sujeto específico de saber - el maestro -, y una institución específica de aplicación y experimentación: la escuela. Con lo cual se le diferencia del concepto de educación, con una esfera mucho más amplia de operación, y con un sentido menos preciso. En segundo lugar, porque "si suprimiéramos una a una las otras ciencias de la educación y dejáramos la Pedagogía y la Didáctica podríamos de todas maneras pensar la enseñanza”. (Zuluaga, 1999, 61) Las obras pedagógicas de Comenio, Pestalozzi, Herbart y Claparède son "unos indicadores significativos acerca de la permanencia de la enseñanza como campo conceptual u operativo donde se concretan los conceptos prácticos del saber pedagógico". (Zuluaga, 1999, 62)

Lo que propone Zuluaga y su equipo de investigación: "Historia de las prácticas pedagógicas en Colombia" es una historia arqueológica de la pedagogía, no para situarla en relación con la ciencia, ni mucho menos para delimitar y legitimar una región de cientificidad. Se trata de recuperarla como una nueva region disciplinaria, como una nueva práctica discursiva, es decir, como saber (saber pedagógico) y como práctica (práctica pedagógica). Es aquí donde la obra de Michel Foucault dotó al Grupo Historia de la Práctica Pedagógica de un lente para entrar en relación con destacados pensadores de la pedagogía ${ }^{8}$ y para observar en los documentos el cruce y las relaciones entre saberes y prácticas. De ahí que en oposición a las perspectivas que defienden las ciencias de la educación, el uso de herramientas arqueológicas busque el establecimiento de relaciones conceptuales en el ámbito del saber pedagógico y de la práctica pedagógica , independientemente de su cientificidad.

Finalmente, el Grupo Historia de la Práctica Pedagógica al plantear recuperar la historicidad de la pedagogía, también, "planteó una problematización histórica de las condiciones de existencia del maestro en el presente, del estatuto marginal de su saber. Esa pregunta, por lo contemporáneo, impulsó la realización de

\footnotetext{
${ }^{8}$ Vives, Comenio, Rousseau, Pestalozzi, Herbart, Claparède, Decroly y Dewey.
} 
una historia de las prácticas y los saberes que fueron condición de posibilidad para la existencia del maestro y de la pedagogía. El problema que sigue vigente y hace volver nuestros ojos al pasado es la condición subalterna del maestro en relación con los otros sujetos que enseñan su saber portando el estatuto de profesor, y no el de maestro, socialmente reconocido como inferior. Aun bajo el respaldo de las Instituciones Formadoras de Docentes, el maestro aprende su oficio como un intelectual subordinado". (Zuluaga, 2004)

\section{REFLEXIONES FINALES}

Hasta el momento presente no existe una historia de las ciencias de la educación planteada en términos comparados entre países de Europa y de Iberoamérica. Las descripciones aquí ofrecidas sobre las culturas francesa, suiza y la colombiana quieren ser entendidas como un primer paso en esa dirección.

Discurso histórico comparado que nos permita hacer visible, con el uso de herramientas arqueológicas, genealógicas y también epistemológicas, las singularidades y diferencias al interior de una unidad territorial. Historia comparada que no haga una apología del poder de las ciencias de la educación al tomar su lugar, en distintas culturas, en la jerarquia propia de la ciencia, sino que posibilite reactivar las particularidades culturales contra el análisis de los discursos locales desde la tiranía de los discursos totalizantes. Historia poscolonial que articule saber y poder, teniendo en cuenta que la circulación de los saberes siempre está articulado a condiciones histórico-políticas. Estas características, que difieren de un país a otro, ponen de relieve que el objeto de los debates y reflexiones epistemológicas sobre las ciencias de la educación no son idénticas sino híbridas. De esta manera, la circulación de las ciencias de la educación, según los ámbitos culturales, actúa en estrategias diferentes, admitiendo, "desplazamientos y reutilizaciones de fórmulas idénticas para objetivos opuestos". (Foucault, 1985, 123) En otras palabras, el discurso de las ciencias de la educación se rearticula en contextos diferentes de aquellos en los que fueron producidos originalmente. El término hibridismo nos hace pensar que los procesos de emergencia e institucionalización de las ciencias de la educación son plurales. Así lo constata Charlot al considerar que el "perfil de las ciencias de la educación difiere según los países o grupos de países: son muy filosóficas en Alemania, más bien psicológicas en Suiza, más sociológicas en Francia y en Gran Bretaña, preocupadas por cuestiones de operacionalidad en Quebec, muy politizadas en Brasil o en Grecia, centradas en el desarrollo de los sistemas escolares en los países del África Negra". (Charlot, 1995, 38)

De ahí la importancia de realizar estudios comparados entre Europa e lberomérica sobre la emergencia de las ciencias de la educación bajo el concepto de "apropiación" propuesto por la profesora colombiana Olga Lucia Zuluaga: “Apropiar es inscribir, en la dinámica particular de una sociedad, cualquier producción técnica o de saber proveniente de otra cultura y generada en condiciones históricas particulares. Apropiar evoca modelar, adecuar, retomar, coger, utilizar, para insertar en un proceso donde lo apropiado se recompone porque entra en una lógica diferente de funcionamiento. Apropiar un saber es hacerlo entrar en coordenadas de la práctica social. Es, por tanto, un proceso que pertenece al orden el saber como espacio donde el conocimiento está accionado por mecanismos de poder y no por la lógica del movimiento de los conceptos en el conocimiento científico. Sin embargo, para historiar un saber apropiado es necesario tomar un campo de conceptos más amplio que el apropiado, con el fin de localizar los recortes, exclusiones, adecuaciones y amalgamas que conlleva tal proceso de institucionalización de ese saber". (Zuluaga, 2004) 


\section{BIBLIOGRAFÍA}

AECSE (Association des enseignants et chercheurs en sciences de l'éducation). Les sciences de l'éducation. Enjeux, finalités et défis. Paris, INRP, 2001.

BUISSON Ferdinand. 'Lecon d'overture du cours de science de l'éducation, faite á la Sorbonne le 3 decembre 1896", in: Revue International de l'enseignement, No. 32(16), 1896, pp. 481-503.

BUISSON Ferdinand. Dictionnaire de Pédagogie et de Instrution Primaire, Paris, Hachette, 1911.

CHARLOT Bernard. "Les sciences de l'éducation en France: une discipline apaisée, une culture commune, un front de recherche incertain", en: HOFSTETTER Rita y SCHNEUWLY Bernard. Le pari des sciences de l'éducation. Belgique, De Boeck \& Larcier, 2001, pp. 147-167.

CHARLOT Bernard. Les sciences de l'éducation, un enjeu, un défi. Paris, ESF, 1995.

CLAPAREDE Edouard. Un Institut des Sciences de l'Education et les besoins auxquelles il répond. Genève, Librairie Kündig, 1912.

COMPAYRE Gabriel. "Pédagogie", en: Grande Encyclopédie, inventaire raissoné des sciences, des lettres et des arts. Citado por: BEST Francine, DEBESSE Maurice y otros. Introducción a la Pedagogía. Barcelona, Oikos-tau, 1972, pp. 58.

COUSINET Roger. La Escuela Nueva. Barcelona, Editorial Miracle, 1967.

DEBESSE Maurice. "Défi aux sciences de l' éducation?", en: L'apport de les sciences fondamentales aux sciences de l'éducation, Paris, EPI, 1976, Volúmen 1.

DEVELAY Michel. "De la ciencia de la educación a las ciencias de la educación", en: Propos sur les sciences de l'éducation. Réflexions épistémologiques. Paris, ESF éditeur, 2001, pp. 11-26.

DOTTRENS Robert y MIALARET Gaston. "El desarrollo de las ciencias pedagógicas y su estado actual", en : BEST Francine. Introducción a la pedagogía, Barcelona, Oikos-Tau Ediciones, 1971.

DURKHEIM Émile. "Pédagogie", EN: Ferdinand Buisson. Dictionnaire de Pédagogie et de Instrution Primaire, Paris, Hachette, 1911, PP. 1538-1543.

FouCAULT Michel. "Erudición y saberes sometidos, Primera Lección 7 de enero de 1976", en: Genealogía del racismo. Madrid, Ediciones la Piqueta, 1992.

FOUCAULT Michel. Arqueología del saber México, Siglo XXI, 1996. Historia de la sexualidad Tomo1, México, Siglo XXI, 1985.

FURTER Pierre. "La educación comparada como geografía de la educación. Cuestiones teóricas sobre la planificación de la regionalización de la enseñanza"., en: PEREYRA Miguel A., MínGUEZ Jesús Garcia y otros. (Compiladores) Globalización y descentralización de los sistemas educativos. Fundamentos para un nuevo programa de la educación comparada. Barcelona, Pomares-Corredor, 1996, pp. 93-115.

GAUTHERIN Jacques. "La ciencia de la educación, disciplina singular, 1883 - 1914", en: CHARLOT Bernard. Les sciences de l'éducation, un enjeu, un défi ESF éditeur, Paris, 1995, páginas, 45 - 54. 
GAUTHERIN Jacqueline. La formation de une discipline universitaire: La science de l' éducation, 1880 - 1914. (Essai, d' histoire social). Thése présentée pour l'obtention du doctorat, sous la direction de Madame le professeur Viviane ISAMBERT-JAMATI. Paris, Université Paris V Rene Descartes, Sciences Humaines, Sorbonne, 1991.

HAMELINE Daniel. "Le statut de la pédagogie", en: Encyclopaedia Universalis. Paris, Tomo XVII, 2002, pp. 590593.

HOFSTETTER Rita y SCHNEUWLY Bernard. "Les débuts de la pédagogie comme discipline universitaire. L'exemple de Genève, 1890 -1916, en: Les Annuelles 8/1997 L'avénement des sciences sociales comme disciplines académiques. Laussane, Antipodes, 1997, pp. 135-171.

Les Sciences de l'éducation en Suisse. Evolution et prospectives.

Berne, Switzerland, CEST (Centre d'études de la science et de la technologie), 2001.

"Pertinence scientifique et pertinence sociale. Mise en perpective de six

rapports nationaux commandités sur la recherche en sciences de l'éducation en Europe", en: Revue Suisse des sciences de l'éducation. Año 22, No (1), 2000, pp 51-91.

Science (s) de l'éducation 19 - 20 siècles. Entre champs professionnels et champs disciplinaires. Bern, Peter Lang, 2002.

MARCEL, Jean-Francois. Les Sciences de l'Education, des recherches, une discipline. Paris, Budapest, Torino, L' Harmattan, 2002.

MEIRIEU Philippe y DeVELAY Michel. Émile, reviens vite... ils sont devenus fous. Paris, ESF éditeur, 1992.

MEIRIEU Philippe. La pédagogie entre le dire et le faire, Paris, ESF éditeur, 1995. Frankenstein educador, Barcelona, Editorial Laertes, 1998.

MialARET Gaston DeBESSE Maurice y otros: Introducción a la Pedagogía, Barcelona, Oikos-tau, 1972.

MiALARET Gaston. Ciencias de la educación. Barcelona, Oikos-tau, 1977.

SÁENZ ObREGón Javier, SALDARRIAGA Oscar, OSPINA Armando. Mirar la infancia: pedagogía, moral y modernidad en Colombia, 1903-1946. Vol. 1, Medellin, Colciencias, Foro Nacional por Colombia, Uniandes, Universidad de Antioquia, 1997.

SALDARRIAGA, Oscar. "La Instrucción Pública en Colombia, 1819-1902: Surgimiento y Desarrollo del Sistema Educativo", en: ZULUAGA Olga Lucia y OSSENBACH SAUTER Gabriela (Compiladoras). Génesis y desarrollo de los Sistemas Educativos Iberoamericanos Siglo XIX. Bogotá, Tomo 1, Colciencias, Universidad de Antioquia, Universidad del Valle, Universidad Pedagógica Nacional, Universidad de los Andes, Grupo Historia de la Práctica Pedagógica, Editorial Magisterio, 2004, pp. 203-287. [En colaboración con Olga Zuluaga, Diego Osorio, Alberto Echeverri, Vladimir Zapata].

SCHRIEWER Jürgen. "La construcción de la pedagogía científica, diferenciación institucional y disciplinar, funciones formativas y praxis educativa de la ciencia universitaria de la educación en Alemania y Francia”, en: Revista de Educación, Madrid, No.( 296), 1991, pp. 137-174 
SOCARRAS José Francisco. Facultades de Educación y Escuela Normal Superior. Su historia y aporte científico, humanístico y educativo. Tunja, Universidad Pedagógica y Tecnológica de Colombia, Ediciones La Rana y el Aguila, 1987.

VeYNE Paul. L'inventaire des différences. Paris, 1976. Citado en: FURTER Pierre. "La educación comparada como geografía de la educación. Cuestiones teóricas sobre la planificación de la regionalización de la enseñanza". en: PeREYRA Miguel A., MíngueZ Jesús Garcia y otros. (Compiladores) Globalización y descentralización de los sistemas educativos. Fundamentos para un nuevo programa de la educación comparada. Barcelona, Pomares-Corredor, 1996, pp. 94.

VIAL Jean. "Pasado y presente de la formación de los maestros", en: BERBAUM Jean. La formación de los enseñantes, Barcelona, Oikos-Tau Ediciones, 1982, pp. 35-91.

ZULUAGA Olga Lucia. El maestro y el saber pedagógico en Colombia, 1821-1848. Medellin, Universidad de Antioquia, 1984.

Pedagogía e Historia: la historicidad de la pedagogía, la enseñanza, Un objeto de saber. Bogotá, Siglo del Hombre Editores, Anthropos, Editorial Universidad de Antioquia, 1999.

"Entre Lancaster y Pestalozzi: Los manuales de formación para la formación de maestros, 1822-1868", en: Revista Educación y Pedagogía. Vol. XIII, No. (29 y 30), Medellin, Facultad de Educación, Universidad de Antioquia, enero-septiembre de 2001, pp. 41-49.

, La Instrucción pública en Colombia, 1845-1868: entre el monopolio y la libertad de enseñanza. El caso de Bogotá. Madrid, Universidad Nacional de Educación a Distancia, 1999. Tesis Doctoral.

, La pedagogía en Francia: precariedad, fragmentación, dispersión, e ilegitimidad. Proyecto de investigación: "El saber pedagógico y las ciencias de la educación”, Medellín, Universidad de Antioquia, CODI, 2004. Documento en proceso de publicación. [En colaboración con Javier Sáenz, Rafael Rios y Sandra Herrera]

Foucault: Una lectura desde la práctica pedagógica, Ponencia presentada en el Seminario Internacional: "pensar de otro modo: usos de Foucault para pensar la educación en Iberoamérica", Santafé de Bogotá, Octubre de 2004. (www.idep.edu.co) 


\title{
Contactar
}

Revista lberoamericana de Educación

\author{
Principal OEI
}

